\title{
Schwarzschild modelling of elliptical galaxies and their black holes
}

\author{
Jens Thomas ${ }^{1,2, \star}$ \\ 1 Universitäts-Sternwarte München, Scheinerstr. 1, D-81679 München, Germany \\ 2 Max-Planck-Institut für extraterrestrische Physik, Giessenbachstr., 85748 Garching, Germany
}

Received 30 May 2005, accepted 11 Nov 2005

Published online later

Key words galaxies: elliptical and lenticular, $\mathrm{cD}$ - galaxies: formation - galaxies: halos - galaxies: kinematics and dynamics - galaxies: structure

\begin{abstract}
This article describes the Schwarzschild orbit superposition method. It is the state-of-the-art dynamical modelling tool for early-type galaxies. Tests with analytic models show that masses and orbital anisotropies of not too face-on galaxies can be recovered with about 15 percent accuracy from typical observational data. Applying Schwarzschild models to a sample of Coma galaxies their dark matter halos were found to be 13 times denser than those of spirals with the same stellar mass. Since denser halos assembled earlier, this result indicates that the formation redshift $1+z_{\text {form }}$ of ellipticals is about two times higher than of spirals. Roughly half of the sample galaxies have halo assembly redshifts in agreement with their stellar-population ages. Galaxies where stars appear younger than the halos show strong phase-space density gradients in their orbital structure, indicative for dissipational evolution and possibly connected with secondary star-formation after the main halo assembly epoch. The importance of considering dark-matter in dynamical models aimed to measure black-hole masses is briefly discussed.
\end{abstract}

(c) 2010 WILEY-VCH Verlag GmbH \& Co. KGaA, Weinheim

\section{Introduction}

Early-type galaxies are characterised by an overall smooth and featureless spheroidal morphology and a dynamically hot system of stellar orbits. This is thought to be the result of a dynamically violent assembly process. However, when and how exactly these galaxies have formed is still not well known. Their mostly old and $\alpha$-enhanced stellar populations imply a relatively short star-formation period in the distant past. The epoch when those stars assembled to form the early-types seen today cannot be directly deduced from stellar population ages. For example, the stars might be born at high redshift, in progenitors that only recently merged into spheroidal galaxies. If these mergers are mostly collisionless (without significant amounts of gas and star-formation), then stellar-population ages do not change, and the main assembly epoch is delayed with respect to the star-formation epoch. In contrast, a spheroidal galaxy formed early in the universe could have grown a disky stellar subcomponent recently, e.g. triggered by a gas-rich minor merger. The main halo assembly would then precede star formation (along the disk).

Pure dark-matter, collisionless $N$-body simulations predict a close relationship between the main assembly redshift of dark matter halos (when, say, half of the mass had been assembled) and their average density. The infall of baryons might change the dark matter density as it enforces an extra gravitational pull on the halo. Still, measuring the dark

\footnotetext{
^ e-mail: jthomas@mpe.mpg.de
}

matter density in elliptical galaxy halos is a valuable tool to gain information about their assembly epoch. In comparison with stellar-population ages it also gives indirect information about the evolution of these systems. According to the above considerations one can expect that galaxies in which stars appear younger than the halos have likely experienced some secondary star-formation episodes. These systems have evolved dissipatively. In contrast, when stars appear older than the halo, this might indicate a more gasless evolution (e.g. by gas-free or collisionless or dry, respectively, mergers).

Since the system of stars in galaxies is collisionless it preserves some information about how the stars have assembled. A dynamically violent formation - characterised by violent relaxation in phase-space - is supposed to result in a highly mixed orbit distribution with strong phase-space density gradients likely being washed out. In contrast, dissipational evolution (e.g. through gas-rich mergers) likely results in disky subsystems with high phase-space density peaks on near-circular orbits. Consequently, the analysis of the orbital structure provides additional information about the assembly mechanism of early-type stellar systems.

In the last years we have collected photometric and kinematic observations for a sample of 19 early-type galaxies in the Coma cluster: 2 central cD galaxies, ten giant ellipticals and seven $\mathrm{S} 0$ or E/S0 galaxies, respectively, with $-18.8 \geq$ $M_{B} \geq-22.26$ (Mehlert et al. 2000; Wegner et al. 2002; Corsini et al. 2008; Thomas et al. 2009a). For all galaxies ground-based and HST photometry are available. Long-slit stellar absorption-line spectra have been taken along at least 
the major and minor axes. In many cases additional data along other position angles has been collected as well. The spectra extend to $1-4 r_{\text {eff }}$, entering the regions where dark matter becomes noticeable.

By means of dynamical models we have measured the dark matter content and orbital structure of these galaxies. The results are summarised below and implications for the formation process of early-type galaxies are discussed.

\section{The Schwarzschild method}

A complete description of a stellar system is provided by its phase-space distribution function $f$, i.e. the density of stars in 6-dim phase-space. Unlike for a collisional gas the distribution function of collisionless dynamical systems like galaxies is not known in advance. However, for steady-state objects Jeans theorem ensures that the phase-space density is constant along individual orbits. Orbits, in turn, are identified by the integrals of motion they respect. Thus, for systems in a steady state the distribution function (DF) reads

$$
f=f\left(I_{1}, \ldots, I_{n}\right),
$$

where $I_{1}, \ldots, I_{n}$ are integrals of motion (e.g. Binney \& Tremaine 1987). The simplest symmetry assumption consistent with the flattening and rotation of elliptical galaxies is that they are axisymmetric. Orbits in typical axisymmetric galaxy potentials respect three integrals of motion: energy $E$, angular momentum along the symmetry axis $L_{z}$ (the rotation axis being parallel to the $z$-axis) and the so-called third integral $I_{3}$ (e.g. Contopoulos 1963). The last integral is usually not known explicitly and the distribution function can thus not be written in terms of elementary functions.

Schwarzschild (1979) introduced an orbit-superposition technique (now called Schwarzschild method) to construct collisionless DFs. In brief, one assumes a trial potential for a given galaxy and composes a library of several thousand orbits. The light distribution and projected kinematics of each orbit are stored. Then, a galaxy model is constructed as the superposition of all orbits. The unknown weight or total amount of light, respectively, of each orbit is chosen to match the model as good as possible with the given constraints (see below). This method corresponds to the approximation $f \approx \sum_{i} f_{i}$, where the $f_{i}$ are single-orbit DFs (Vandervoort 1984; Thomas et al. 2004). The accuracy of the method only depends on the density of the orbit grid.

The Schwarzschild method provides very general dynamical models. In contrast to Jeans models there is no necessity for any a priori restriction upon the orbital structure. Moreover, Schwarzschild models are easily constructed to be everywhere positive in phase space (i.e. physically meaningful) - a property that is not guaranteed in Jeans models. The main challenge is to ensure that the orbit library is representative for all orbital shapes.

In axisymmetric potentials it is straight forward to sample the energy $E$ and the angular momentum $L_{z}$. The issue related to our ignorance about $I_{3}$ is overcome by a systematic sampling of orbital initial conditions, which implicitly guarantees the inclusion of all orbital shapes. More specifically, orbits with given $E$ and $L_{z}$ but different $I_{3}$ follow distinct invariant curves in appropriate surfaces of section (SOS). Launching orbits at constant $E$ and $L_{z}$ from various initial starting points on a grid in such SOSs ensures a representative sampling of the unknown $I_{3}$ (Thomas et al. 2004).

In practice, one goes through the following steps to construct a Schwarzschild model of a galaxy:

- The photometry of the galaxy is deprojected to obtain the $3 \mathrm{~d}$ light distribution $\nu$.

- A trial mass distribution $\rho$ is set up, combining the various mass components, e.g.

$$
\rho=\Upsilon \times \nu+\rho_{\mathrm{DM}}+M_{\mathrm{BH}} \times \delta(r) .
$$

Here, $\Upsilon$ is the stellar mass-to-light ratio (typically assumed to be radially constant in ellipticals), $\rho_{\mathrm{DM}}$ is the dark matter density (see below) and $M_{\mathrm{BH}}$ represents a supermassive central black hole. Given $\rho$, the gravitational potential follows by solving Poisson's equation and an orbit library can be assembled.

- The orbits are superposed and the orbital weights that best match with the observations are determined. In our implementation this is done by maximising

$$
S-\alpha \chi^{2}
$$

where $\chi^{2}$ quantifies deviations between observed and modelled kinematics (the observed light profile is used as a boundary condition in the maximisation). The function

$$
S=-\int f \ln f \mathrm{~d}^{3} r \mathrm{~d}^{3} v
$$

is the Boltzmann entropy of the orbit distribution and $\alpha$ is a regularisation parameter (Richstone \& Tremaine 1988; see also below).

- The parameters determining the potential (e.g. $\Upsilon$, halo parameters, $M_{\mathrm{BH}}$ ) are systematically varied and the final best-fit is obtained from a $\chi^{2}$ analysis.

Various implementations of this method exist for spherical (Romanowsky et al. 2003), axisymmetric (Cretton et al. 1999; Cappellari et al. 2006; Chanamé, Kleyna \& Van der Marel 2008; Valluri, Merritt \& Emsellem 2004; Gebhardt et al. 2003; Thomas et al. 2004) as-well as triaxial potentials (van den Bosch et al. 2008). Gebhardt et al. (2003) measured supermassive black-holes in the centres of galaxies (ignoring any contribution of dark matter). Cappellari et al. (2006) modelled a subsample of the SAURON galaxies, again ignoring dark matter because their data cover only the inner regions $<r_{\text {eff }}$.

Beyond $r_{\text {eff }}$ dark matter becomes important in earlytype galaxies. The Coma sample is by now the only larger sample of generic (e.g. flattened and rotating) ellipticals that has been modelled with Schwarzschild's method including dark matter. Previous attempts to measure the dark matter content of early-types via stellar dynamics focussed on round and non-rotating systems and assumed spherical symmetry (Kronawitter et al. 2000; Gerhard et al. 2001; Magorrian \& Ballantyne 2001). 


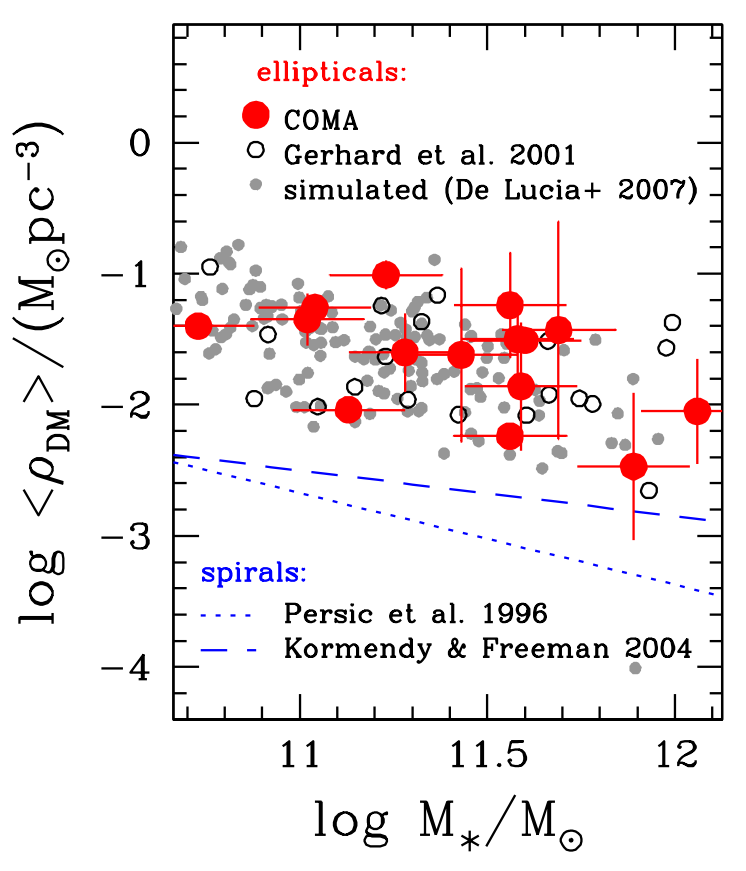

Fig. 1 Dark matter density $\left\langle\rho_{\mathrm{DM}}\right\rangle$ (averaged within $2 r_{\text {eff }}$ ) versus stellar mass $M_{*}$. Red dots: Coma early-type galaxies; open circles: round and non-rotating galaxies from Gerhard et al (2001); small dots: semi-analytic galaxy formation models (De Lucia \& Blaizot 2007). Blue lines: spiral galaxies.

In the Coma galaxies we probed for two different parametric halo profiles: (1) logarithmic halos with a constantdensity core and asymptotically constant circular velocity and (2) so-called NFW-profiles which are good fits to dark matter halos in cosmological $N$-body simulations (Navarro, Frenk \& White 1996). The majority of Coma galaxies are better fit with logarithmic halos, but the significance over NFW halo profiles is marginal (Thomas et al. 2007b).

\section{Tests of the method}

A unique feature of our Schwarzschild models is the incorporation of orbital phase-space volumes $V$ (Thomas et al. 2004). Together with the total amount of light $w$ on the orbit it allows to calculate the phase-space density $f=w / V$ on each orbit. This offers several new applications. Firstly, one can project analytic phase-space DFs $f_{\text {analytic }}$ through orbit libraries. In this case, the orbital weights are not determined from a fit to photometric and kinematic constraints, but directly from the analytic $\mathrm{DF}$, i.e. the weight $w_{i}$ of orbit $i$ reads $w_{i}=f_{\text {analytic }} \times V_{i}$. The projected kinematics, spatial density profile, and intrinsic velocity dispersion profiles of the so-constructed orbit superposition can be compared with direct phase-space integrations of $f_{\text {analytic }}$. Both methods agree very well (Thomas et al. 2004), confirming that the orbit sampling is representative.
In addition, mock data from analytic model galaxies can be used to measure how accurate the orbital DF can be reconstructed. In this Monte-Carlo approach photometric and kinematic data with spatial resolution and coverage similar to real data are simulated and modelled exactly as real galaxies. For the Coma data we have shown that with the appropriate choice of the regularisation parameter $\alpha$ the orbital structure and mass distribution of not too face-on galaxies can be recovered with an accuracy of about 15 percent (Thomas et al. 2005). Similar tests have been presented in Cretton et al. (1999), Krajnović et al. (2005) and Siopis et al. (2009).

The input models for the above tests had the same symmetry as the dynamical models (axial symmetry). Isophotal twists and kinematic misalignment suggest that at least the most massive early-type galaxies are slightly triaxial. In order to explore the systematic errors arising from too restrictive symmetry assumptions, we applied our models also to mock data sets created from collisionless $N$-body binary disk merger remnants. These remnants are strongly triaxial in the central, box-orbit dominated regions (Jesseit, Naab $\&$ Burkert 2005). While the central mass measured with axisymmetric models then underestimates the true mass on average (depending on projection angle and box-orbit content) the enclosed mass within $r_{\text {eff }}$ is still recovered mostly with better than 20 percent accuracy unless for highly flattened, face-on systems (Thomas et al. 2007a).

\section{The dark matter density and assembly epoch of early-type galaxies}

Fig. 11 shows dark matter densities $\left\langle\rho_{\mathrm{DM}}\right\rangle$ as a function of stellar mass. Stellar masses $M_{*}=\Upsilon \times L$ of early-types are derived from the best-fit (dynamical) stellar mass-to-light ratio $\Upsilon$ and the observed total luminosity $L$. Dark matter densities $\left\langle\rho_{\mathrm{DM}}\right\rangle$ are averaged within $2 r_{\text {eff }}$.

The dark matter densities of Coma ellipticals (from axisymmetric modelling) and round and non-rotating galaxies (from spherical models) match well. With increasing stellar mass dark matter densities tend to decrease. Spiral galaxy dark matter densities are also shown in Fig. 1)(Persic, Salucci \& Stel 1996a,b; Kormendy \& Freeman 2004, respectively). Stellar masses for spirals have been derived using the Tully-Fisher and stellar-mass Tully-Fisher relations of Bell \& De Jong (2001) (cf. Thomas et al. 2009a for details). As in early-types, dark matter densities in spirals decrease with increasing stellar mass. Most important, the dark matter in ellipticals is about 13 times denser than in spirals of the same stellar mass (compared at the same $B$-band luminosity, early-type galaxies have 7 times denser halos than late-types).

Finally, Fig. 1]also includes semi-analytic galaxy formation models of De Lucia \& Blaizot (2007). They are in good agreement with the observations in Coma galaxies. This is surprising because the $N$-body cosmological simulation underlying the semi-analytic models was performed without 


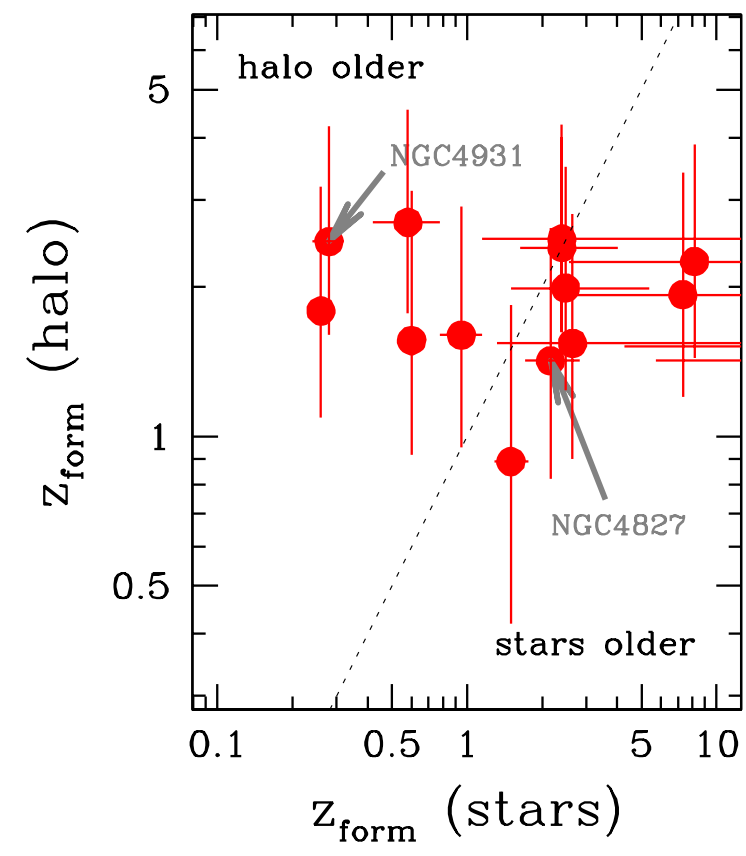

Fig. 2 Halo assembly redshifts (y-axis) versus starformation redshift ( $\mathrm{x}$-axis) for Coma early-type galaxies. The one-to-one relation is indicated by the dotted line. The phase-space DFs of the two galaxies NGC4827 and NGC4931 are shown in Fig. 4

baryon dynamics. Thus, either the net effect of baryons on dark matter around early-types in the given mass interval vanishes or there is some discrepancy between the semianalytic models and the observations. Note that baryons can also cause a halo expansion (e.g. by dynamical friction).

The light distribution in late-type galaxies is less centrally concentrated than in early-types. Then, even if the net effect of baryons on elliptical galaxy halos would be negligible, the stronger gravitational pull in early-type galaxy halos could still contribute to the observed overdensity of dark matter in ellipticals relative to spirals. Within the adiabatic contraction approximation this can at most explain a factor of two between average halo densities around ellipticals and spirals, respectively (Thomas et al. 2009a). The remaining over-density indicates that ellipticals have assembled earlier - at a time where the universe was denser.

The simplest analytic models, as well as pure dark matter cosmological $N$-body simulations predict a scaling of the average dark matter density $\left\langle\rho_{\mathrm{DM}}\right\rangle$ with halo-assembly redshift $z_{\text {form }}$ of $\left\langle\rho_{\mathrm{DM}}\right\rangle \propto\left(1+z_{\text {form }}\right)^{3}$ (e.g. Gerhard et al. 2001). Accordingly, the density contrast between elliptical and spiral galaxy halos translates into $1+z_{\text {form }}$ being about two times higher for ellipticals than for spirals. Absolute assembly redshifts can only be estimated with an additional assumption upon the formation redshift of spirals. Supposed that spirals assemble typically at $z_{\text {form }} \approx 1$ (at higher redshifts spirals become rare, e.g. Conselice et al. 2005) then
Coma early-types have assembled around $z_{\text {form }} \approx 2-3$. These dark-matter based formation redshifts are shown in Fig. 2 against stellar-population ages. In about one third of Coma galaxies the stars appear to be younger than the halos but many galaxies are consistent with equal assembly and star-formation redshifts.

\section{The orbital structure of early-type galaxies}

As outlined above a galaxy in which the stars appear to be younger than the halo is a candidate dissipative system, while objects in which stars are equally old or older than the halo have likely formed monolithically or through mostly collisionless mergers. In dissipative systems one would expect a high phase-space density on near-circular disk orbits while the phase-space DF of systems which have undergone violent relaxation should lack strong phase-space density gradients.

Classically, the orbital structure is measured in terms of anisotropy parameters, i.e. ratios of intrinsic velocity dispersions. Let $f$ denote the phase-space DF of a galaxy, then the intrinsic dispersions $\sigma_{i j}$ read

$$
\begin{aligned}
& \sigma_{i j}^{2}=\frac{1}{\rho} \int f\left(v_{i}-\overline{v_{i}}\right)\left(v_{j}-\overline{v_{j}}\right) \mathrm{d}^{3} v, \\
& \overline{v_{i}}=\frac{1}{\rho} \int f v_{i} \mathrm{~d}^{3} v .
\end{aligned}
$$

In the following we assume $i, j \in\{R, \phi, z\}$, where $z$ is the symmetry axis (cylindrical coordinates). The unordered kinetic energy along coordinate direction $i$ is

$$
\Pi_{i i}=\int \rho \sigma_{i i}^{2} \mathrm{~d}^{3} r
$$

and the anisotropy in the velocity dispersions can be quantified by $\beta \equiv 1-\Pi_{z z} / \Pi_{R R}$ and $\gamma \equiv 1-\Pi_{\phi \phi} / \Pi_{R R}$ (Cappellari et al. 2007). An isotropic system has $\beta=\gamma=0$.

Fig. 3. shows $\beta$ and $\gamma$ versus intrinsic ellipticity $\epsilon$. In observed galaxies $\beta>0$ and, on average, $\gamma \approx 0$, but with significant scatter. The range of anisotropies found in Coma early-types is similar as in SAURON galaxies but the Coma galaxies do not exhibit a trend of increasing $\beta$ with increasing $\epsilon$ as observed by Cappellari et al (2007). Neither the inclusion of dark matter in the Coma models nor regularisation can explain this difference (Thomas et al. 2009b). Instead it most likely reflects differences in the selections of the two samples (Thomas et al. 2009b).

In order to clarify the relationship between the classical anisotropy and the intrinsic orbital structure two prototypical orbital compositions are shown by the lines in Fig. 3. (1) The case of flattening by rotation, i.e. by extra-light on near-circular orbits. Circular orbits mostly contribute to the kinetic energy in $\phi$ direction and $\gamma$ becomes negative. The more, the flatter the galaxy is (dashed lines). (2) The dotted lines show toy models with maximum entropy 1 . Without any other conditions the maximisation of Boltzmann's

\footnotetext{
1 For both toy models 75 percent of the light was distributed on prograde orbits; cf. Thomas et al. (2009b) for details.
} 


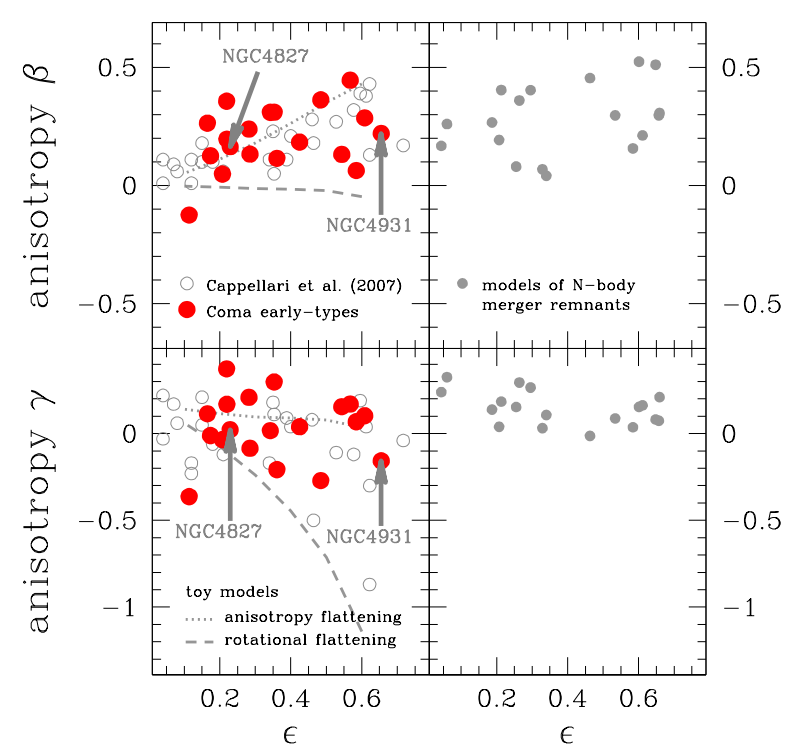

Fig. 3 Anisotropy $\beta$ and $\gamma$ versus intrinsic ellipticity $\epsilon$ for observed galaxies (left-hand panels) and $N$-body binary disk merger remnants (right-hand panels). Dotted lines: maximum-entropy toy-models, dashed lines: 2I models (details in the text). The phase-space DFs of the two galaxies NGC4827 and NGC4931 are shown in Fig.4.

entropy yields a flat DF $f=$ const. This is altered as soon as the luminosity distribution is used as a boundary condition. Still, maximising the entropy yields, in a sense, the smoothest phase-space DF compatible with a given density profile. It turns out that the classical notion of flattening by anisotropy (i.e. a suppression of vertical versus horizontal energy, with isotropy between $R$ and $\phi$ ) is closely related to the maximisation of entropy. Accordingly, systems along the maximum-entropy line, while having varying anisotropy $\beta$, are similar in that their phase-space distribution functions are smooth.

The phase-space DFs of two Coma galaxies shown in Fig. 4 (both galaxies are flagged by the arrows in Figs. 2 and 3). The two galaxies have similar $\beta$ and $\gamma$ but different intrinsic flattening: NGC4827 is an E2 early-type while NGC4931 is highly flattened. In addition, in NGC4827 stars are about as old as the halo while the stars in NGC4931 appear younger than its halo.

In the upper panels of Fig. 4 spheroidal orbits are highlighted. Disk orbits are highlighted in the lower panels. We identify spheroidal orbits by $|\vartheta|_{\max }>70^{\circ}$, where $\vartheta$ is the angle between a point on the orbit and the equatorial plane of the galaxy. So-defined spheroidal orbits come close to the galaxy's minor-axis and contrast disk orbits, which are instead selected by $|z|_{\max }<r_{\text {eff }} / 4$, where $z$ is the vertical height of a point on the orbit with respect to the equatorial plane.

Fig. (4)shows that phase-space densities on spheroidal orbits are similar in both galaxies. Moreover, in NGC4827 the

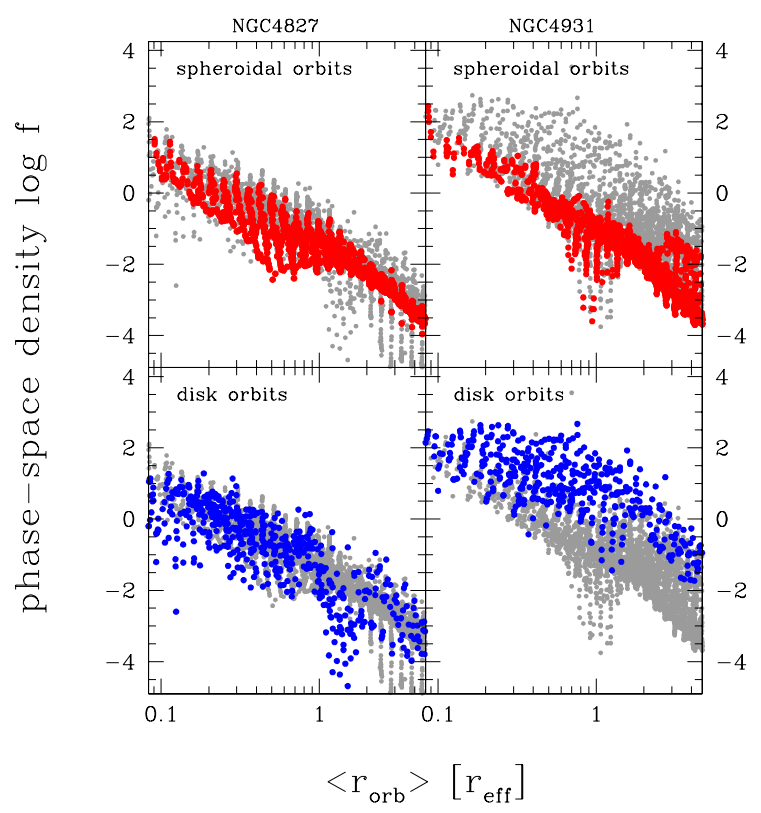

Fig. 4 Phase-space distribution function $f$ for the two galaxies NGC4827 (left-hand panels) and NGC4931 (righthand panels). Each dot represents the phase-space density on a single orbit (in solar masses per cubed kpc and cubed $\mathrm{km} / \mathrm{s}$ ) plotted against the mean orbital radius. In the top panels spheroidal orbits are highlighted; in the bottom panel disk orbits are highlighted (details in the text). Only prograde orbits are shown.

phase-space densities of disk orbits are comparable to those of spheroidal orbits. Yet, the stellar density on disk orbits in NGC4931 is up to two orders of magnitude higher than on spheroidal orbits. These findings support the interpretation that galaxies left to the one-to-one relation in Fig. 2 (stars younger than halo) are dissipative systems, while galaxies close to the one-to-one relation or right of it are systems which formed in a dynamically violent process and have smoother phase-space DFs. A more detailed investigation of phase-space densities will be presented in Thomas et al. (in preparation).

The right-hand panels of Fig. 3 display dynamical models of collisionless $N$-body binary disk merger remnants. Mock data sets with similar spatial resolution and coverage as for Coma galaxies were constructed from projections along the three principal axes of six remnants selected representatively from the $N$-body sample of Naab \& Burkert (2003). While the distribution of $\beta$ versus $\epsilon$ is like in observed galaxies, none of the analysed merger remnants has $\gamma<0$. In view of the above discussion this is plausible since galaxies with $\gamma<0$ are likely dissipative systems (large energy in $\phi$ caused by extra-light on circular orbits), while the analysed $N$-body merger simulations are gas-free. 


\section{Summary}

The Schwarzschild technique is the state-of-the-art tool to model early-type galaxies. For typical observational data masses and orbital anisotropies can be recovered with about 15 percent accuracy (for not too face-on systems). We have applied Schwarzschild models to 19 Coma early-type galaxies to measure the dark matter content and distribution of stellar orbits. By today, it is the largest sample of generic early-type galaxies with dynamical models including dark matter. Dark matter densities in early-types are larger than in spirals of the same luminosity or mass. Extra gravitational pulling by the more concentrated baryons in ellipticals is not sufficient to explain this over-density. Instead, the formation redshift $1+z_{\text {form }}$ of early-types is about two times higher than of spirals. Under the assumption $z_{\text {form }} \approx 1$ for spirals the Coma early-types have formed around $z_{\text {form }} \approx$ $2-3$. Observed dark matter densities are in good agreement with recent semi-analytic galaxy formation models. In about half of the sample galaxies halo assembly redshifts match with stellar population ages. In galaxies where stars appear younger than the halo, the orbit distribution indicates dissipational evolution (i.e. strong phase-space density peaks on near-circular disk orbits). This suggest that these galaxies had some secondary star-formation after the main halo assembly epoch.

When modelling the very central regions of early-types to study their supermassive central black-holes it is important to include dark matter in the models, even if the central parts itself are not dominated by dark matter. Neglect of the halo can result in an overestimation of the stellar mass-tolight ratio which subsequently leads to an overestimation of the central stellar mass. The latter is degenerate with the black-hole mass and neglect of dark matter might then yield a too small black-hole. This has been illustrated for M87, where the black-hole mass more than doubled after including dark matter in the models (Gebhardt \& Thomas 2009). The effect is supposed to be strongest for the most massive galaxies since their light profiles are shallow. Further galaxies have to be modelled in order to establish if the inclusion of dark matter in black-hole models can reduce the discrepancy between the $\approx 10^{9} M_{\odot}$ solar mass black-holes in the most massive nearby galaxies and the $\approx 10^{10} M_{\odot}$ solar mass black-holes in high-redshift quasars.

\section{References}

Bell, E.F., \& De Jong, R.S.: 2001, ApJ , 550, 212

Binney, J., \& Tremaine, S.: 1987, Galactic Dynamics (Princeton: Princeton University Press)

Cappellari, M., et al.: 2006, MNRAS , 366, 1126

Cappellari, M. et al.: 2007, MNRAS, 379, 418

Chanamé, J., Kleyna, J., \& van der Marel, R.: 2008, ApJ , 682,841

Conselice, C.J., Blackburne, J.A., \& Papovich, C.: 2005, ApJ , 620, 564
Contopoulos, G.: 1963, AJ, 68, 1

Corsini, E.M., Wegner, G., Saglia, R.P., Thomas, J., Bender, R., Thomas, D.: 2008, ApJS , 175, 462

Cretton, N., de Zeeuw, P.T., van der Marel, R.P., \& Rix, H.W.: 1999, ApJS , 124, 383

De Lucia, G., \& Blaizot, J.: 2007, MNRAS , 375, 2

Gebhardt, K., et al.: 2003, ApJ , 583, 92

Gebhardt, K., \& Thomas, J.: 2009, ApJ, 700, 1690

Gerhard, O.E., Kronawitter, A., Saglia, R.P., Bender, R.: 2001, AJ , 121, 1936

Jesseit, R., Naab, T. \& Burkert, A.: 2005, MNRAS, 360, 1185

Kormendy, J., \& Freeman, K.C.: 2004, in IAU Symp. 220, Dark Matter in Galaxies, ed. S.D. Ryder et al. (San Francisco: ASP), 377

Krajnović, D., Cappellari, M., Emsellem, E., McDermid, R.M., de Zeeuw, P.T.: 2005, MNRAS, 357, 1113

Kronawitter, A., Saglia, R.P., Gerhard, O.E., Bender, R.: 2000, A\&AS, 144, 53

Magorrian, J., Ballantyne, D.: 2001, MNRAS, 322, 702

Mehlert, D., Saglia, R.P., Bender, R., Wegner, G.: 2000, A\&AS, 141, 449

Mehlert, D., Thomas, D., Saglia, R.P., Bender, R., Wegner, G.: 2003, A\&A, 407, 423

Naab, T. \& Burkert, A.: 2003, ApJ, 597, 893

Navarro, J.F., Frenk, C.S., \& White, S.D.M.: 1996, ApJ , 462, 563

Persic, M., Salucci, P., \& Stel, F.: 1996a, MNRAS , 281, 27

Persic, M., Salucci, P., \& Stel, F.: 1996b, MNRAS , 283, 1102

Richstone, D. O., \& Tremaine, S.: 1988, ApJ, 327, 82

Romanowsky, A.J., Douglas, N.G., Arnaboldi, M., Kuijken, K., Merrifield, M.R., Napolitano, N.R., Capaccioli, M., Freeman K.C.: 2003, Sci, 301, 1696

Schwarzschild, M.: 1979, ApJ , 232, 236

Siopis, C. et al.: 2009, ApJ , 693, 946

Thomas, J., Saglia, R.P., Bender, R., Thomas, D., Gebhardt, K., Magorrian, J., Richstone, D.: 2004, MNRAS , 353, 391

Thomas, J., Saglia, R.P., Bender, R., Thomas, D., Gebhardt, K., Magorrian, J., Corsini, E.M., Wegner, G.: 2005, MNRAS , 360, 1355

Thomas, J., Jesseit, R., Naab, T., Saglia, R. P., Burkert, A., \& Bender, R.: 2007a, MNRAS , 381, 1672

Thomas, J., Saglia, R.P., Bender, R., Thomas, D., Gebhardt, K., Magorrian, J., Corsini, E.M., Wegner, G.: 2007b, MNRAS , 382, 657

Thomas, J., Saglia, R.P., Bender, R., Thomas, D., Gebhardt, K., Magorrian, J., Corsini, E.M., Wegner, G.: 2009a, ApJ , 691, 770

Thomas, J., et al.: 2009b, MNRAS , 393, 641

Valluri, M., Merritt, D., \& Emsellem, E.: 2004, ApJ , 602, 66

Vandervoort, P.O.: 1984, ApJ, 287, 475

Wegner, G., Corsini, E. M., Saglia, R. P., Bender, R., Merkl, D., Thomas, D., Thomas, J., Mehlert, D.: 2002, A\&A, 
395,753

van den Bosch, R.C.E., van de Ven, G., Verolme, E.K., Cap-

pellari, M., \& de Zeeuw, P.T.: 2008, MNRAS , 385, 647 